\title{
Anxiety and behavior: mother and child in situation of preventive pediatric dental care.
}

\author{
Isabela G Lima (IC), Juliana B Warlet (IC), Rosana F Possobon (PQ)
}

\begin{abstract}
These researches aimed to assess children behavior during preventive pediatric dental care and degrees of maternal anxiety, on general and specific dental treatment situation. Children on dental treatment, even when it is preventive and, therefore, with less potential to cause pain and discomfort, must receive dentist support, to enable better adaptation to dental treatment, and also help to have lower degrees of maternal anxiety on this kind of situation.
\end{abstract}

Key words: anxiety, dental fear, uncooperative behavior.

\section{Introduction}

Some researches point to a relation between maternal anxiety and children notcollaborating behavior during dental treatment. These researches aimed to assess children behavior during preventive pediatric dental care and degrees of maternal anxiety, on general and specific dental treatment situation, and identify preventive procedures with bigger potential to generate uncooperative behavior. The sample had 110 children, aged between 12 and 60 months, and their mothers. These children had oral health only needing preventive treatment, without any physical, sensory and motor disease; attend on a session of preventive dental program from Cepae - FOP - Unicamp. The data about children behavior was collected from their clinical records and anxiety and dental fear from their mothers were measured using IDATEtrace/condition, DAS e DFS. The data was analyzed on distribution and frequency.

\section{Results and Discussion}

Results shown that the procedure that elicited bigger crying frequency was biofilm disclosure $(10 \%)$ realized by dentist, and was also the procedure that demanded greater dentist effort, once that children was low collaborating $(9,1 \%)$. A considerable portion among mothers shown high anxiety degrees, according to the instruments used on research. (DAS: $16,4 \%$ on moderate degree and $3,6 \%$ on exacerbated; DFS: $67,3 \%$ on medium degree and $4,5 \%$ on high level). When mothers are anxious during their child treatment, (IDATE-condition), 15,5\% of mothers present high levels, once that this frequency was closer to the high levels of anxiety founded to high maternal degrees (IDATE-Trace: $14,5 \%)$.

\section{Conclusions}

Children on dental treatment, even when it is preventive and, therefore, with less potential to cause pain and discomfort, must receive dentist support, to enable better adaptation to dental treatment, and also help to have lower degrees of maternal anxiety on this kind of situation.

\section{Acknowledgement} concession.

We thank PIBIC-CNPq for the studentship

Tomita, L. M., Costa Jr., A. L., Moraes, A. B. A. de. Ansiedade materna manifestada durante o tratamento odontológico de seus filhoes (2007)

Possobon, R.F., Moraes, A.B.A., Costa Junior, A.L. \& Ambrosano, G.M.B. O comportamento de crianças durante atendimento odontológico. Psicologia: Teoria e Pesquisa. 2003; 19: 5-64. 